\title{
Autonomic nervous system activity and the risk of nosocomial infection in critically ill patients with brain injury
}

\author{
Mathijs R. Wirtz ${ }^{1,2,3^{*}+}$ C, Jiri Moekotte ${ }^{1,2+}$, Kirsten Balvers ${ }^{1,2,3}$, Marjolein M. Admiraal ${ }^{1,2}$, Jean-Francois Pittet ${ }^{5}$, \\ Joe Colombo ${ }^{6}$, Brant M. Wagener ${ }^{5}$, J. Carel Goslings ${ }^{4}$ and Nicole Juffermans ${ }^{1,2}$
}

${ }^{*}$ Correspondence:

m.r.wirtz@amsterdamumc.nl

${ }^{\dagger}$ Mathijs R. Wirtz and Jiri

Moekotte have contributed

equally to the manuscript

${ }^{1}$ Department of Intensive

Care Medicine, Onze

Lieve Vrouwe Gasthuis

and Amsterdam University

Medical Centers, Amsterdam

The Netherlands

Full list of author information is available at the end of the article

\begin{abstract}
Purpose: Nosocomial infection contributes to adverse outcome after brain injury. This study investigates whether autonomic nervous system activity is associated with a decreased host immune response in patients following stroke or traumatic brain injury (TBI).
\end{abstract}

Methods: A prospective study was performed in adult patients with TBI or stroke who were admitted to the Intensive Care Unit of our tertiary university hospital between 2013 and 2016. Heart rate variability (HRV) was recorded daily and assessed for autonomic nervous system activity. Outcomes were nosocomial infections and immunosuppression, which was assessed ex vivo using whole blood stimulations with plasma of patients with infections, matched non-infected patients and healthy controls.

Results: Out of 64 brain injured patients, 23 (36\%) developed an infection during their hospital stay. The ability of brain injured patients to generate a host response to the bacterial endotoxin lipopolysaccharides (LPS) was diminished compared to healthy controls $(p<0.001)$. Patients who developed an infection yielded significantly lower TNF-a values ( $86 \mathrm{vs} 192 \mathrm{pg} / \mathrm{mL}, p=0.030$ ) and a trend towards higher IL-10 values (122 vs $84 \mathrm{pg} / \mathrm{mL}, p=0.071$ ) following ex vivo whole blood stimulations when compared to patients not developing an infection. This decreased host immune response was associated with altered admission HRV values. Brain injured patients who developed an infection showed increased normalized high-frequency power compared to patients not developing an infection ( 0.54 vs $0.36, p=0.033$ ), whereas normalized low-frequency power was lower in infected patients ( 0.46 vs $0.64, p=0.033$ ).

Conclusion: Brain injured patients developing a nosocomial infection show parasympathetic predominance in the acute phase following brain injury, reflected by alterations in HRV, which parallels a decreased ability to generate an immune response to stimulation with LPS.

Keywords: Autonomic nervous system, Brain injury, Immunosuppression, Nosocomial infection author(s) and the source, provide a link to the Creative Commons licence, and indicate if changes were made. The images or other third party material in this article are included in the article's Creative Commons licence, unless indicated otherwise in a credit line to the material. If material is not included in the article's Creative Commons licence and your intended use is not permitted by statutory regulation or exceeds the permitted use, you will need to obtain permission directly from the copyright holder. To view a copy of this licence, visit http:// creativecommons.org/licenses/by/4.0/. 


\section{Introduction}

Stroke and traumatic brain injury (TBI) result in high rates of infectious complications, with subsequent high mortality rates [1,2]. Patients admitted to the intensive care unit (ICU) are vulnerable to nosocomial infection, for example due to iatrogenic factors such as indwelling catheters and risk of aspiration prior to intubation [3]. However, these risk factors do not fully explain the disproportional higher infection rates in brain injured patients when compared to the general ICU population [1, $4,5]$. Brain injury-mediated immunosuppression is a recognized clinical phenomenon in brain injured patients $[2,4,5]$.

Following neurotrauma, a disruption in the well-balanced interplay between the nervous and immune system occurs within hours of the insult and can last for several weeks [6]. Cerebral inflammatory responses produce pro-inflammatory cytokines and an influx of immune cells, causing neural cell death and brain damage [7]. Possibly, this initial inflammatory burst exhausts the immune system causing peripheral immunosuppression. The mechanism by which this occurs is not fully understood, but activation of the autonomic nervous system may contribute to immunosuppression following brain injury [7, 8]. A non-invasive method to investigate autonomic nervous system activity is through heart rate variability (HRV) analysis, describing interval changes between successive heartbeats [9]. Cardiac rhythm is regulated by the autonomic nervous system through alterations in sympathetic and parasympathetic tone resulting in changes in HRV. These alterations may be physiologic, but can also be the result of (critical) illness. HRV therefore represents a complex reflection of autonomic responsiveness of the cardiovascular system to changes in autonomic outflow and provides information on autonomic nervous system activity and sympathovagal balance. Increased parasympathetic nervous system activity correlates with immunosuppression and mortality in brain injured patients $[10,11]$. This occurs through vagal nerve stimulation activating the cholinergic anti-inflammatory pathway, resulting in decreased systemic pro-inflammatory cytokine production, such as TNF- $\alpha[8]$.

This study aimed to elucidate the role of the autonomic nervous system in the risk of developing infections following brain injury. The primary aim of this study was to determine whether parasympathetic surges are associated with immunosuppression and nosocomial infection rates in brain injured patients. A secondary aim was to elucidate temporal trends in autonomic nervous system activity along with patterns of inflammatory response profiles associated with brain injury-mediated immunosuppression.

\section{Materials and methods}

This prospective study was performed from March 2013 to July 2016 in our tertiary university hospital in the Netherlands. This study was approved by the ethics committee of the Amsterdam University Medical Centers. Patients were included following informed consent from next of kin and the study was conducted according to the principles of the Declaration of Helsinki and adhering to good clinical practice guidelines and the Medical Research Involving Human Subjects Act (WMO). 


\section{Population}

All patients $\geq 18$ years old were eligible for inclusion if they suffered from TBI or stroke (ischemic or hemorrhagic) and required admission to the intensive care unit. Exclusion criteria included expected death within $1 \mathrm{~h}$ of admission, burn or inhalation injury or emergency thoracotomy or cardiopulmonary resuscitation with chest compressions before ICU arrival. Patients with known pregnancy, receiving immunosuppressive medication prior to admission, with a known do-not-resuscitate order and patients already enrolled in a concurrent ongoing interventional randomized clinical trial were also excluded.

\section{Heart rate variability recordings}

Patients were hemodynamically monitored during their ICU or medium care stay. For HRV analysis, the heart rate of patients was recorded from the patient bedside monitor with specialized software from our hospital's technical department (HeartRateMonitor3, Academic Medical Center, Amsterdam, The Netherlands). This software determines the position of the $\mathrm{R}$ peaks in the patient's electrocardiography (ECG) at a sampling rate of $1000 \mathrm{~Hz}$. Ten-minute epochs of the ECG were recorded directly after admission to the ICU and subsequently four times daily during the admission period. Daily recorded 10-min epochs were pooled together to calculate a daily mean to prevent circadian variation.

\section{Blood samples}

Blood samples were collected on the admission day and 24 and $72 \mathrm{~h}$ thereafter (days 0,1 and 3 ). The blood samples were centrifuged for $10 \mathrm{~min}$ at 1750 relative centrifugal force (RCF) at $18{ }^{\circ} \mathrm{C}$. The upper two-thirds of the plasma was centrifuged again to obtain platelet-free plasma and stored as $250-\mu \mathrm{L}$ aliquots at $-80^{\circ} \mathrm{C}$.

\section{Definition of infection}

Infection criteria were adopted from the MARS project (Molecular Diagnosis and Risk Stratification of Sepsis) [12], which has provided criteria for infection classification that were modified from the Centers for Disease Control (CDC) and International Sepsis Forum (ISF) criteria. All available clinical, microbiological, and radiological evidence of infections was collected. Infections were scored based in their infection source and certainty of diagnosis, resulting in definite, probable and possible infections. The MARS criteria for infections can be found in the online Additional file 1, 'criteria for diagnosed infections'.

\section{Antibiotic management}

Selective digestive tract decontamination (SDD), consisting of parenteral and enteral antimicrobials, was administered to patients with an expected ICU stay of $>3$ days. This includes a 4-day regime of intravenous cefotaxime. Also, an enteral nonabsorbable suspension containing polymyxin, tobramycin and amphotericin B was administered through a nasogastric tube four times a day. Orabase paste was applied four times a day to the oropharyngeal mucosa. Furthermore, antibiotics chosen based on local protocol and expert opinion were started empirically when an infection was suspected. 


\section{Whole blood stimulation}

Whole blood stimulations were performed as described before [13]. Briefly, heparinanticoagulated blood from a healthy volunteer was collected. $500 \mu \mathrm{L}$ whole blood samples were diluted in a 1:1 ratio with RPMI (Roswell Park Memorial Institute, Buffalo, USA), supplemented with glutamine $0.3 \mathrm{~g} / \mathrm{L}$ and lipopolysaccharide (LPS E. coli; O111:B4 Ultrapure SIGMA, $1 \mathrm{ng} / \mathrm{mL}$ ). Subsequently, $100 \mu \mathrm{L}$ of plasma from either infected or non-infected patients was added. Whole blood stimulated with LPS without the addition of patient plasma served as a positive control. Whole blood buffered with RPMI without the addition of patient plasma or LPS served as a negative control. The samples were then incubated for $24 \mathrm{~h}$ at $5 \% \mathrm{CO}_{2}$ at $37^{\circ} \mathrm{C}$. After incubation, the samples were centrifuged $\left(1200 \mathrm{RCF}\right.$ at $18{ }^{\circ} \mathrm{C}$ for $\left.10 \mathrm{~min}\right)$ and the upper two-thirds of the plasma was collected and stored at $-20^{\circ} \mathrm{C}$.

\section{ELISA}

TNF- $\alpha$ and IL-10 levels were measured in the supernatant collected after the whole blood stimulations by enzyme-linked immunosorbent assays (ELISA) according to the manufacturer's instructions (R\&D Systems, Abingdon, United Kingdom).

\section{Outcome}

The primary outcome of our study were nosocomial infections, autonomic nervous system activity and the extent of immunosuppression, defined as the outcome of ex vivo whole blood stimulations. Secondary outcomes included temporal trends in heart rate variability and immunosuppression, duration of ventilation, length of hospital and ICU stay and 28-day mortality.

\section{Statistical analyses}

HRV recordings were analyzed with HRV algorithms in MATLAB software (MathWorks, Natick, USA) adhering to international standards [14]. The rationale behind the algorithms is described in more detail in the online Additional file 1. Normalized units of high-frequency power (HFnu) as a reflection of parasympathetic activity and normalized units of low-frequency power (LFnu) as a reflection of sympathetic activation were used to assess autonomic activity [14]. Also low-frequency:high-frequency (LF:HF) ratio was analyzed, as this ratio is considered to reflect either the sympathovagal balance or sympathetic nervous system activity [10].

All patients with definite or probable infections were selected for ex vivo whole blood stimulation tests. These patients were compared to non-infected patients, matched for age and APACHE II scores.

Distribution of variables was examined for normality using the KolmogorovSmirnov test. Continuous variables are expressed as mean and standard deviation (SD) or median and interquartile range (IQR) and were analyzed using the Student's $t$-test or the Mann-Whitney $U$ test, depending on the distribution. Categorical factors are expressed as proportions and were analyzed by Chi-square testing. Testing between infected patients, non-infected patients and controls in ex vivo whole blood 
Table 1 Baseline characteristics of brain injured patients

\begin{tabular}{|c|c|c|c|}
\hline & All patients $(n=64)$ & Infected $(n=23)$ & Non-infected $(n=41)$ \\
\hline \multicolumn{4}{|l|}{ Main characteristics } \\
\hline Age (years, $n, \%)$ & $51(19.3)$ & $52(18.2)$ & $50(20.1)$ \\
\hline Male gender $(n, \%)$ & $47(73 \%)$ & $15(65 \%)$ & $32(78 \%)$ \\
\hline \multicolumn{4}{|l|}{ Reason for ICU admission ( $n, \%)$} \\
\hline TBI & $50(78 \%)$ & $18(78 \%)$ & $32(78 \%)$ \\
\hline iCVA & $9(14 \%)$ & $2(9 \%)$ & $7(17 \%)$ \\
\hline hCVA & $5(8 \%)$ & $3(13 \%)$ & $2(5 \%)$ \\
\hline \multicolumn{4}{|l|}{ Admission injury/illness severity scores } \\
\hline ISS (mean, SD) & $24(11)$ & $26(12)$ & $23(11)$ \\
\hline AIS thorax $\geq 3(n, \%)$ & $13(26 \%)$ & $5(28 \%)$ & $8(25 \%)$ \\
\hline AIS abdomen/pelvis $\geq 3(n, \%)$ & $2(4 \%)$ & $1(6 \%)$ & $1(3 \%)$ \\
\hline AlS extremity $\geq 3(n, \%)$ & $4(8 \%)$ & $0(0 \%)$ & $4(13 \%)$ \\
\hline AIS external $\geq 3(n, \%)$ & $0(0 \%)$ & $0(0 \%)$ & $0(0 \%)$ \\
\hline GCS (median, IQR) & $7(3-9)$ & $7(3-8)$ & $6(3-9)$ \\
\hline SAPS 2 (mean, SD) & $43.7(11.3)$ & $45.5(9.3)$ & $42.7(12.3)$ \\
\hline APACHE II (median, IQR) & $19(16-23)$ & $19(17-21)$ & $19(15-23)$ \\
\hline \multicolumn{4}{|l|}{ Comorbidity } \\
\hline Prehospital beta-blocker use $(n, \%)$ & $10(16 \%)$ & $4(17 \%)$ & $6(15 \%)$ \\
\hline Hypertension ( $n, \%)$ & $14(22 \%)$ & $5(22 \%)$ & $9(22 \%)$ \\
\hline $\operatorname{CVA}(n, \%)$ & $6(9 \%)$ & $3(13 \%)$ & $3(7 \%)$ \\
\hline Cardiac pathology $(n, \%)$ & $11(17 \%)$ & $3(13 \%)$ & $8(20 \%)$ \\
\hline Diabetes mellitus $(n, \%)$ & $6(9 \%)$ & $2(9 \%)$ & $4(10 \%)$ \\
\hline $\operatorname{COPD}(n, \%)$ & $4(6 \%)$ & $1(4 \%)$ & $3(7 \%)$ \\
\hline Alcohol abuse $(n, \%)$ & $5(8 \%)$ & $2(9 \%)$ & $3(7 \%)$ \\
\hline \multicolumn{4}{|l|}{ Admission clinical parameters } \\
\hline Heart rate (bpm, mean, SD) & $80(17)$ & $83(15)$ & $79(18)$ \\
\hline SBP (mmHg, mean, SD) & $132(29)$ & $132(37)$ & $133(24)$ \\
\hline Temperature $\left({ }^{\circ} \mathrm{C}\right.$, mean, $\left.\mathrm{SD}\right)$ & $35.6(1.2)$ & $35.5(1.3)$ & $35.7(1.2)$ \\
\hline \multicolumn{4}{|l|}{ Admission lab parameters } \\
\hline $\mathrm{Hb}(\mathrm{mmol} / \mathrm{l}$, median, IQR) & $7.9(6.7-8.5)$ & $7.2(6.3-8.2)^{*}$ & $8.0(7.5-8.8)$ \\
\hline WCC $(\times 10 \wedge 9 / /$, mean SD) & $11.8(4.7)$ & $11.6(5.8)$ & $11.9(4.0)$ \\
\hline CRP (mg/l, median, IQR) & $3.8(0.7-6.7)$ & $3.8(2-7.4)$ & $4.9(0.6-7.8)$ \\
\hline
\end{tabular}

Data are presented as mean standard deviation, median and interquartile range or absolute number and percentage ICU intensive care unit, TBI traumatic brain injury, iCVA ischemic cerebrovascular accident, $h C V A$ hemorrhagic cerebrovascular accident, ISS Injury Severity Score, AIS Abbreviated Injury Scale, GCS Glasgow Coma Score, SOFA Sequential Organ Failure Assessment Score, SAPS 2 Simplified Acute Physiology Score 2, APACHE II Acute Physiology and Chronic Health Evaluation 2 score, $M I$ myocardial infarction, $A F$ atrial fibrillation, COPD chronic obstructive pulmonary disease, SBP systolic blood pressure, $\mathrm{Hb}$ hemoglobin, WCC white cell count, CRP C-reactive protein

*Significantly different from non-infected group

+ Cardiac pathology includes MI and AF, as well as cardiac valve pathology and other (paroxysmal) rhythm disorders

stimulation tests was done with the one-way ANOVA test. $p$ Values less than 0.05 were considered statistically significant.

\section{Results}

Of 1712 screened patients, of these, 151 patients were eligible for inclusion, of whom 75 patients were excluded because no informed consent was obtained, they were expected to die within $1 \mathrm{~h}$ after admission, or they were discharged within $24 \mathrm{~h}$ of ICU admission. 


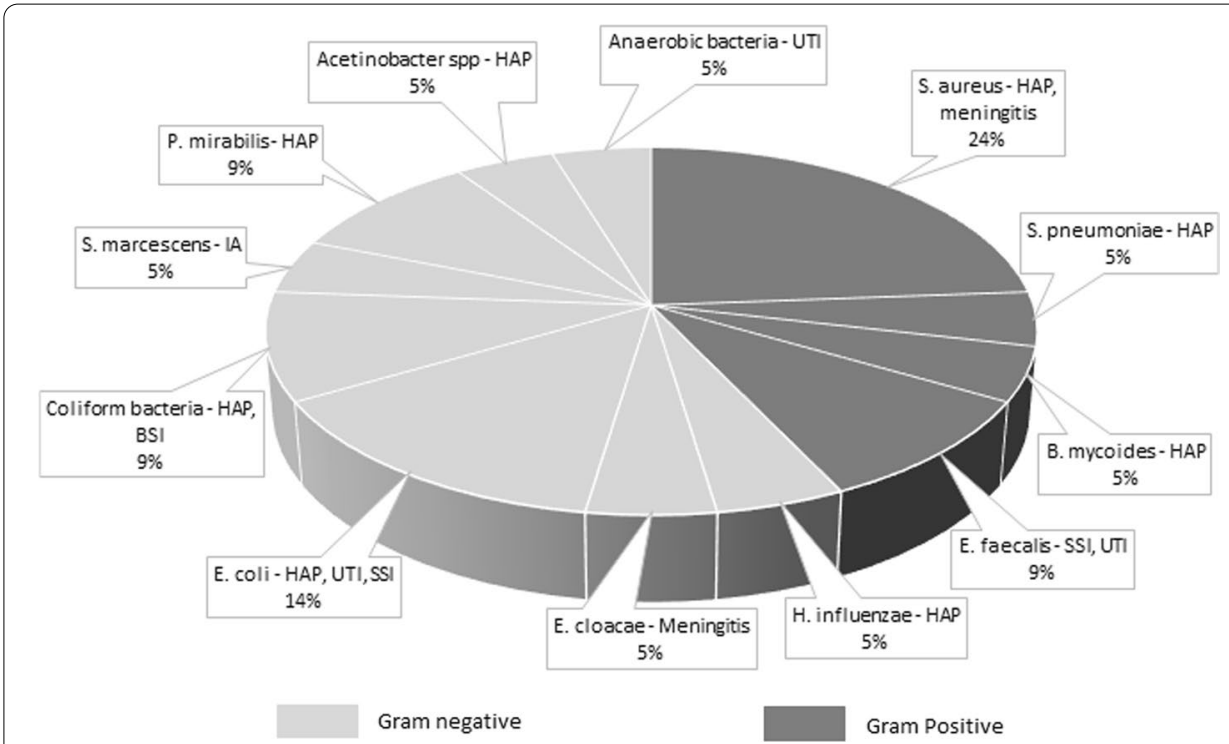

Fig. 1 Pathogens found in positive microbiology cultures. HAP hospital acquired pneumonia, SS/ surgical site infection, UTI urinary tract infection, BS/ blood stream infection, IA intracranial abscess

A total of 12 patients were missed for inclusion due to logistic difficulties. A total of 64 brain injured patients were included. Patient demographics and baseline characteristics are displayed in Table 1. Patients were $51 \pm 19$ years old and $73 \%$ were male. 50 patients (78\%) suffered from TBI (with or without subsequent hemorrhagic stroke), 9 patients (14\%) from ischemic stroke and 5 patients (8\%) were admitted with hemorrhagic stroke. Median GCS was 7 on emergency department admission and patients were critically ill and severely injured, as reflected by a median APACHE II score of 19 and a mean ISS score of 24 .

\section{Infections}

Baseline characteristics between patients who did and did not develop an infection were similar, with the exception of hemoglobin levels being lower in infected patients (Table 1). The type and probability of infections among included patients are presented in Additional file 1: Table 1. A total of 23 patients (36\%) developed at least 1 infection during their hospital stay. Infections in 8 patients were classified as definite, in 5 patients as probable and in 10 as possible. The total number of infections was 28 , as 4 patients developed multiple infections. Pneumonia was most frequently observed $(n=16)$, followed by urinary tract infections $(n=4)$ and intracranial infections $(n=4)$. Other observed infections include primary blood stream infections and surgical site infections. Two-thirds of infected patients developed an infection during their initial ICU stay, while the others developed an infection after transfer to the ward. Staphylococcus aureus was most frequently cultured (25\%, Fig. 1). Patients who developed an infection did so after a median of 6 days (IQR 3-12) after admission. More invasive procedures were performed in infected patients compared to patients not developing an infection (see Table 2). No difference was seen in the use or duration of antibiotic treatment. 
Table 2 Treatment on ICU and outcome

\begin{tabular}{|c|c|c|c|c|}
\hline & All patients $(n=64)$ & Infected $(n=23)$ & $\begin{array}{l}\text { Non- } \\
\text { infected } \\
(n=41)\end{array}$ & $p$-value \\
\hline \multicolumn{5}{|l|}{ Invasive procedures } \\
\hline Surgery $(n, \%)$ & $38(59)$ & $17(74)$ & $21(51)$ & 0.076 \\
\hline $\begin{array}{l}\text { Amount of surgical procedures ( } n \text {, median, } \\
\text { IQR) }\end{array}$ & $1(0-1)$ & $1(0-3)$ & $1(0-1)$ & 0.006 \\
\hline Tracheostomy $(n, \%)$ & $10(16)$ & $8(35)$ & $2(5)$ & 0.002 \\
\hline Intracranial device $(n, \%)$ & $20(31)$ & $12(52)$ & $8(20)$ & 0.007 \\
\hline $\begin{array}{l}\text { Duration of Intracranial device (days, } \\
\text { median, IQR) }\end{array}$ & $0(0-2)$ & $1(0-6)$ & $0(0-0)$ & 0.005 \\
\hline Central venous catheter $(n, \%)$ & $26(41)$ & $12(52)$ & $14(34)$ & 0.159 \\
\hline $\begin{array}{l}\text { Duration of central venous catheter (days, } \\
\text { median, IQR) }\end{array}$ & $0(0-5)$ & $2(0-8)$ & $0(0-3)$ & 0.103 \\
\hline \multicolumn{5}{|l|}{ Treatment during ICU/CMC stay } \\
\hline Antibiotics ( $n, \%)$ & $48(75)$ & $19(83)$ & $29(71)$ & 0.292 \\
\hline $\operatorname{SDD}(n, \%)$ & $44(69)$ & $18(78)$ & $26(63)$ & 0.219 \\
\hline Other than SDD $(n, \%)$ & $37(58)$ & $16(70)$ & $21(51)$ & 0.154 \\
\hline Duration of antibiotics (days, median, IQR) & $5(1-7)$ & $5(2-9)$ & $4(0-7)$ & 0.126 \\
\hline Vasopressor ( $n, \%)$ & $35(55)$ & $17(74)$ & $18(44)$ & 0.021 \\
\hline $\begin{array}{l}\text { Duration of vasopressor use (days, median, } \\
\text { IQR) }\end{array}$ & $1(0-2)$ & $1(0-5)$ & $0(0-2)$ & 0.016 \\
\hline Beta-blocker $(n, \%)$ & $12(19)$ & $7(30)$ & $5(12)$ & 0.073 \\
\hline $\begin{array}{l}\text { Duration of beta-blocker use (days, median, } \\
\text { IQR) }\end{array}$ & $0(0-0)$ & $0(0-2)$ & $0(0-0)$ & 0.066 \\
\hline Mechanically ventilated $(n, \%)$ & $61(95)$ & $23(100)$ & $38(93)$ & 0.184 \\
\hline Duration of ventilation (days, median, IQR) & $3(2-7)$ & $6(2-11)$ & $2(2-5)$ & 0.042 \\
\hline \multicolumn{5}{|l|}{ Outcome } \\
\hline Onset of (first) infection (days, median, IQR) & NA & $6(3-12)$ & NA & NA \\
\hline ICU stay (days, median, IQR) & $4.5(3-9)$ & $8(3-14)$ & $4(2-6.5)$ & 0.011 \\
\hline $\begin{array}{l}\text { Total length of hospital stay (days, median, } \\
\text { IQR) }\end{array}$ & $12(6-26)$ & $24(10-44)$ & $9(5-17)$ & 0.001 \\
\hline \multicolumn{5}{|l|}{ Discharge location ( $n, \%)$} \\
\hline Home & $20(31)$ & $4(17)$ & $16(39)$ & 0.073 \\
\hline Another hospital & $17(27)$ & $8(35)$ & $10(24)$ & 0.375 \\
\hline Nursing home & $14(22)$ & $7(30)$ & $7(17)$ & 0.215 \\
\hline \multicolumn{5}{|l|}{28 -day mortality $(n, \%)$} \\
\hline All causes & $12(19)$ & $4(17)$ & $8(19)$ & 0.835 \\
\hline Brain injury & $10(8)$ & $3(13)$ & $7(16)$ & 0.670 \\
\hline MOF & $2(3)$ & $1(4)$ & $1(2)$ & 0.674 \\
\hline
\end{tabular}

Data are presented as median and interquartile range or absolute number and percentage

$S D D$ selective decontamination of the digestive tract, ICU intensive care unit

Prehospital use of beta-blockers was also similar ( 15 vs $15 \%, p=0.80$ ), as was its use at the day of admission to the ICU ( 5 vs $9 \%, p=0.55)$.

\section{Cytokine production after whole blood stimulation}

Cytokine production in response to ex vivo stimulation with LPS was measured in 12 patients with definite or probable infections and compared to non-infected matched controls as well as healthy controls (see Fig. 2). Brain injured patients had a significant 


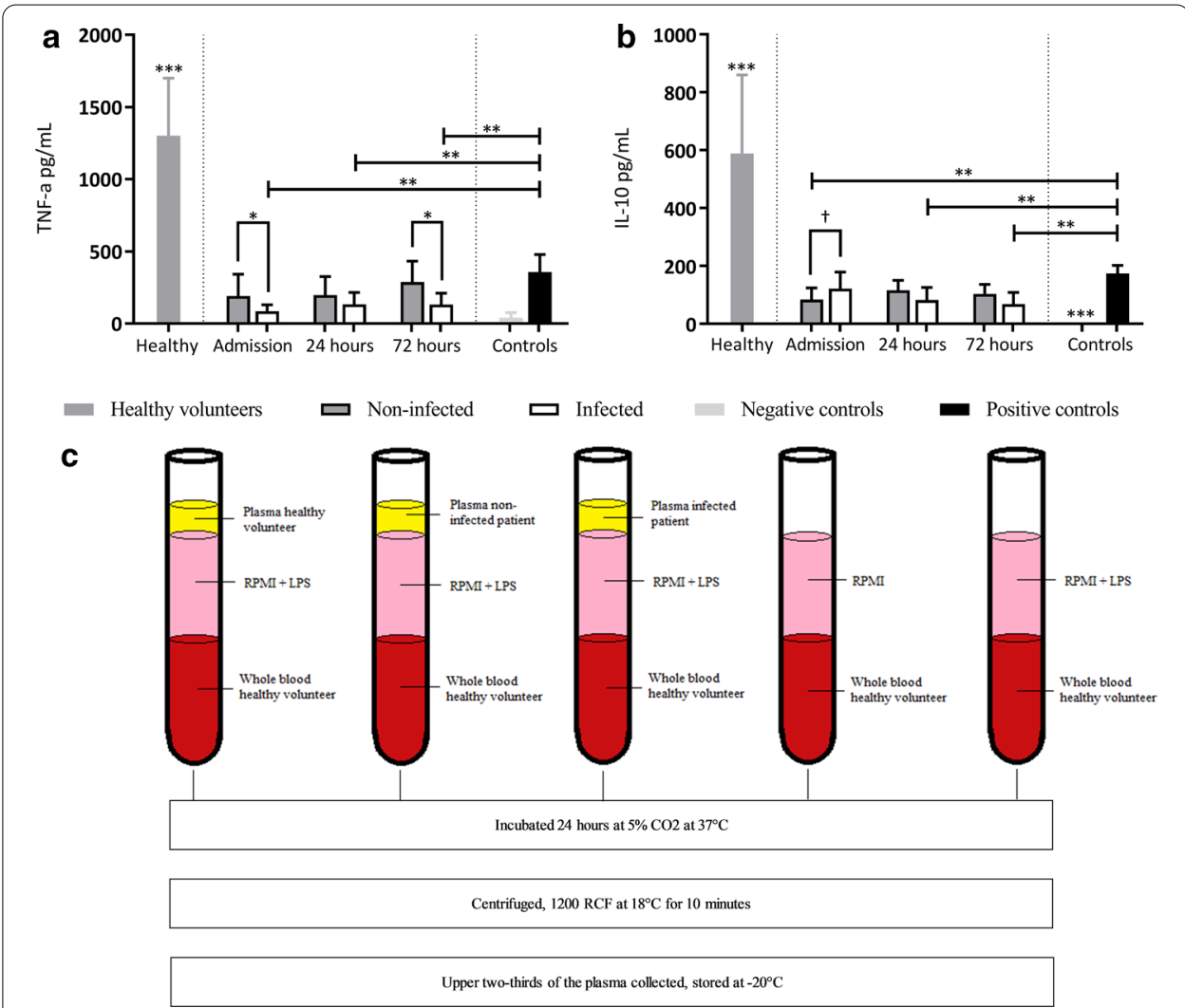

Fig. 2 Cytokine production after ex vivo whole blood stimulation. Cytokine production after whole blood stimulation with LPS for samples collected on admission, and 1 and 3 days after admission to the ICU. Values are represented as mean and standard deviation. a TNF-a production measured after $24 \mathrm{~h}$ of incubation time. $\mathbf{b} \mathrm{IL}-10$ production measured after $24 \mathrm{~h}$ of incubation time. $\mathbf{c}$ Experimental set-up of whole blood stimulations. Healthy = whole blood stimulations with LPS and incubated with plasma samples of healthy volunteers. Non-infected $=$ whole blood stimulations with LPS incubated with plasma samples of patients without an infection. Infected = whole blood stimulations with LPS incubated with plasma samples of patients with a probable or definite infection. Negative controls = whole blood buffered with RPMI without LPS and without the addition of patient plasma. Positive control $=$ whole blood stimulation with LPS without the addition of patient plasma. $\mathrm{tp}<0.10 ;{ }^{*} \mathrm{p}<0.05 ;{ }^{* *} \mathrm{p}<0.01$; ${ }^{* * *} \mathrm{p}<0.001$ compared to all other measurements

lower ability to generate TNF- $\alpha$ and IL-10 production when compared to healthy controls (all $p<0.001$ ). On admission to the ICU, patients who developed an infection during their hospital stay had lower TNF- $\alpha$ production in response to LPS compared to non-infected patients ( $86 \mathrm{vs} 192 \mathrm{pg} / \mathrm{mL}, p=0.030$ ). This difference was also apparent three days after admission (113 vs $287 \mathrm{pg} / \mathrm{mL}, p=0.024$ ).

Compared to patients without an infection, a trend towards higher IL-10 values at baseline was found in infected patients (122 vs $84 \mathrm{pg} / \mathrm{mL}, p=0.071$ ).

\section{Heart rate variability}

Results of heart rate variability analysis of the acute phase following admission after brain injury are shown in Table 3. At ICU admission, patients who acquired an infection later during their hospital stay had significantly lower normalized low-frequency power (LFnu, 0.46 vs $0.64, p=0.033$ ) accompanied with higher normalized high-frequency 
Table 3 Admission HRV parameters

\begin{tabular}{|c|c|c|c|}
\hline HRV parameter & Infected $(n=9)$ & Not infected $(n=19)$ & $p$-value \\
\hline LF (msec ${ }^{2}$, median, IQR) & $53(5-121)$ & $117(58-339)$ & 0.029 \\
\hline HF (msec ${ }^{2}$, median, IQR) & $72(27-90)$ & $72(22-177)$ & 0.572 \\
\hline TP (msec ${ }^{2}$, median, IQR) & $349(97-750)$ & $353(247-1218)$ & 0.446 \\
\hline LF:HF ratio (median, IQR) & $0.93(0.43-2.45)$ & $1.82(1.33-4.36)$ & 0.065 \\
\hline LFnu (mean, SD) & $0.46(0.24)$ & $0.64(0.18)$ & 0.033 \\
\hline HFnu (mean, SD) & $0.54(0.24)$ & $0.36(0.18)$ & 0.033 \\
\hline
\end{tabular}

Data are presented as mean and SD or as median and IQR

LF low frequency, HF high frequency, TP total power, LF:HF ratio ratio between low and high frequency, LFnu normalized units of low-frequency power, HFnu normalized units of high-frequency power

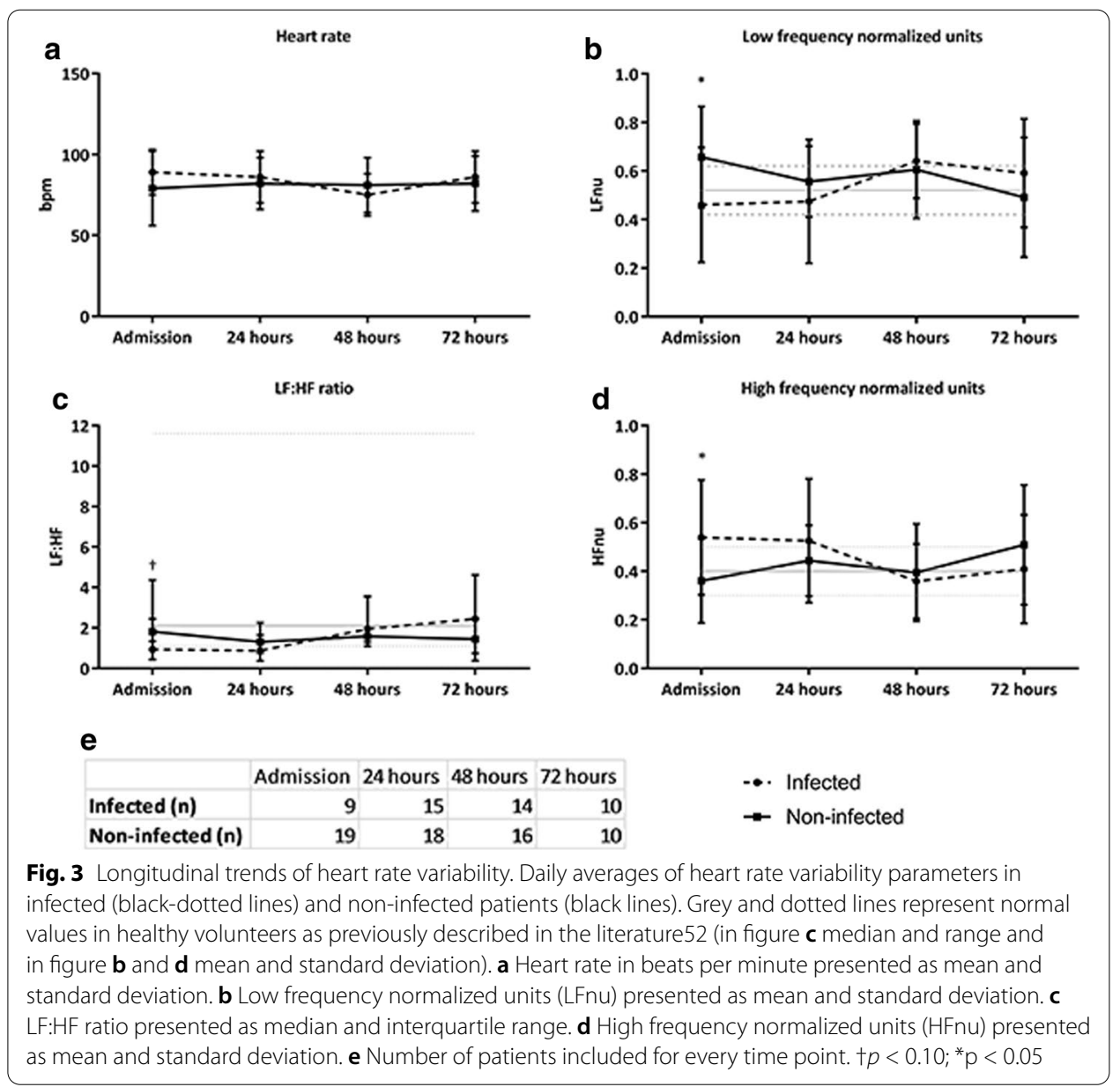

power (HFnu, 0.54 vs $0.36, p=0.033$ ) when compared to patients who did not acquire an infection. Furthermore, a trend was found towards a lower LF:HF ratio in infected patients compared to non-infected patients ( 0.93 vs $1.82, p=0.065$ ).

Figure 3 shows the longitudinal patterns of HRV after brain injury. Following the acute phase, patients not developing an infection showed a decrease in LFnu and LF:HF and an increase in HFnu in the first $24 \mathrm{~h}$, while these values remained stable in patients who developed an infection. After $24 \mathrm{~h}$, there was no significant difference between the 
groups. Patients developing an infection during their stay in the hospital had a median LF:HF ratio below reference values of healthy volunteers, as well as a higher HFnu in the first $24 \mathrm{~h}$ after admission to the ICU. Patients without an infection showed values within these reference ranges.

\section{Outcome}

Duration of mechanical ventilation was significantly longer in patients who developed an infection compared to those who did not (6 vs 2 days, $p=0.042$ ). More often, these patients required the use of vasopressors ( $74 \mathrm{vs} 44 \%, p=0.021$ ) and required these for a longer time period ( 1 vs 0 day, $p=0.016$ ). Also, hospital length of stay was significantly prolonged when patients developed an infection ( 24 vs 9 days, $p=0.001$ ), as was ICU stay ( 8 vs 4 days, $p=0.011$ ). However, 28 -day mortality rates did not differ between infected and non-infected patients (17 vs $19 \%, p=0.835$, see Table 2).

\section{Discussion}

Out of 64 brain injured patients, 36\% developed an infection. All brain injured patients showed a decreased ability to generate an immune response to bacterial LPS stimulation ex vivo compared to healthy controls. When compared to non-infected patients, patients developing an infection had a reduced immunoresponsiveness, reflected by decreased TNF- $\alpha$ values after ex vivo bacterial stimulation. The development of an infection was associated with a parasympathetic surge at ICU admission. At $72 \mathrm{~h}$ after injury, parasympathetic activity decreased, whereas TNF- $\alpha$ production in response to a bacterial stimulus remained abrogated.

Despite the fact that $75 \%$ of patients received antibiotic treatment during their ICU stay, with $53 \%$ already starting on admission, $36 \%$ of patients developed an infection during their hospital stay. This incidence is similar to findings in previous studies [1,2]. Of note, most pathogens found in positive microbiology cultures in infected patients in our study were covered by SDD prophylaxis. Also, the number of patients receiving antibiotic treatment did not differ between patients developing an infection and those who did not, even after correcting for the use of SDD. This shows that antibiotic (prophylactic) treatment may not effectively prevent infections in these patients. In brain injury patients, evidence for the use of prophylactic antibiotics is scarce and somewhat conflicting, with most studies showing no effect of prophylactic antibiotics in preventing pneumonia $[4,15]$.

Immunosuppression following brain injury is a phenomenon that is increasingly recognized. Our study shows that reduced immunoresponsiveness increases susceptibility to post-injury infection. The fact that a reduced immunoresponsiveness is apparent directly after injury suggests that it is a cause and not the result of an infection. This is supported by a recent study in which an immunosuppressive phenotype has been found in lung tissue of brain injured mice that were inoculated with Streptococcus pneumonia, even when mice were inoculated two months after the primary injury [16]. This stresses the fact that immunosuppression following brain injury is a severe complication with long lasting consequences. 
Patients developing an infection in our study required more and longer supportive care and stayed in the ICU longer. However, as patients showed a parasympathetic surge along with immunosuppression on admittance to the ICU, we believe these procedures are a consequence of the infection, rather than the cause. This is underlined by the fact that $77 \%$ of procedures were performed after the infection was diagnosed.

Significant autonomic regulation dysfunction in brain injured patients has been reported, with lower levels of overall HRV when compared to healthy controls [10, 17]. Our data demonstrate an autonomic shift towards parasympathetic dominance preceding the occurrence of infection, reflected by higher HFnu values. This parasympathetic surge corroborated with a systemic immune suppression as reflected by a decreased ability to generate TNF- $\alpha$ in ex vivo stimulated plasma samples. Previously published data confirmed that parasympathetic activity correlated strongly with lower plasma TNF- $\alpha$ levels [10]. Thereby parasympathetic activity may up-regulate the brain's immune system at the expense of the body's immune system, as was shown in septic trauma patients [18]. Also, it has been shown that brain injury leads to increased activity of vagal nerve parasympathetic fibers, which in turn leads to downregulation of TNF- $\alpha$ production through the cholinergic pathway without affecting IL-10 production [19]. What is more, a study evaluating HRV in the acute phase after ischemic stroke found that increased vagal activity (increased HFnu) was predictive of the development of subacute infections [20].

Our findings may have potential clinical consequences. HRV, in particular HFnu, may distinguish patients at risk of acquiring an infection and serve as an indicator to initiate therapy. Several potential therapies intervening in autonomic regulation have been described. Trials on counteracting autonomic nervous system activity by beta-blockade show conflicting results [21-23]. A recent systematic review in TBI patients showed that infections occurred more often in patients receiving beta-blockade [24]. In our study, total in-hospital administration of beta-blockers was similar between infected and noninfected patients, although a trend towards more and longer beta-blocker use was found in patients who developed an infection. The use of beta-blocking agents may result in higher parasympathetic activity, as measured by HRV [25]. In our study parasympathetic hyperactivity preceded development of an infection and was not related to beta-blocker administration. Thereby, our results do not support the use of beta-blockers in brain injured patients already in a parasympathetic hyperactive state at admission to the ICU.

Several therapies targeting the cholinergic pathway have also been suggested in brain injury patients [26]. Research with animal models demonstrated that inhibition of cholinergic signaling, by vagotomy or by using acetylcholine receptor-deficient mice, enabled reversal of the observed immune hyporesponsiveness and prevented pneumonia after stroke [27]. Preclinical studies investigating the administration of muscarinic- and nicotinic-acetylcholine receptor antagonists showed a benefit in neural survival by preventing the effects of acute elevation of acetylcholine in parasympathetic hyperactivation. What is more, pharmacological inhibition of the nicotinic-acetylcholine receptor reduced lung injury and mortality in mice with a post-stroke pneumonia [28]. However, the effect of these agents needs further research, specifically focusing on the effect of these agents on the parasympathetic surge in the acute phase after brain injury and the relation with immune functioning and infection rates. 


\section{Limitations}

We did not retain raw electrocardiography data, nor did we record respiratory variability, rendering it more difficult to isolate the parasympathetic activity signal from the sympathetic activity signal [29]. Also, only explorative analysis could be performed without correcting for multiple comparisons given our small cohort. For the same reason no subgroup analysis of TBI and stroke patients could be performed. Therefore, heterogeneity may have influenced our results. Additional replication studies using larger cohorts of patients will be necessary to confirm our findings. Furthermore, uniform infection criteria were used in this study, however some patients may have received an unjustified diagnosis of infection, especially patients in the possible infection category. Lastly, the use of HRV has some drawbacks. Due to the large heterogeneity and poor description of the methodology of studies using HRV analysis and the lack of a uniform protocol for its effectuation, comparisons with contemporary literature may be hampered.

\section{Conclusion}

Infection occurs frequently after brain injury. Hyperactivity of the parasympathetic nervous system in the acute phase after injury may predispose patients to the development of an infection, due to a decreased systemic immunoresponsiveness. Whether high parasympathetic outflow, as reflected by the corresponding HRV indices, might thus function as an early indicator of patients at risk for infection needs further investigation. Results of this study may stimulate research aiming at improving the autonomic balance in order to reduce infection rates.

\section{Supplementary information}

Supplementary information accompanies this paper at https://doi.org/10.1186/s40635-020-00359-3.

Additional file 1: Criteria for diagnosed infections.

\section{Acknowledgements}

None.

Authors' contributions

Study concept and design: NJ, JC, JG, BW, JP. Acquisition, analysis, or interpretation of data: MW, JM, KB. Drafting of the manuscript: MW, JM. Critical revision of the manuscript for important intellectual content: MW, JM, KB, MA, JP, JC, BW, JG, NJ. Statistical analysis: MW, JM, MA, NP. All authors read and approved the final manuscript.

\section{Funding}

Not applicable.

Availability of data and materials

The data that support the findings of this study are available from the corresponding author, MW, upon reasonable request.

\section{Code availability}

Not applicable.

Ethics approval and consent to participate

This study was performed with approval of the ethics committee of the Amsterdam University Medical Centers. The study was conducted according to the principles of the Declaration of Helsinki and adhering to good clinical practice guidelines and the Medical Research Involving Human Subjects Act (WMO). Written informed consent was obtained from a legal representative or from the patient in case the patient was deemed awake and adequate. 


\section{Competing interests}

Prof. Colombo was medical director and part-owner of Ansar Medical technologies, Inc. when this study was performed. Dr. Wagener and Prof. Pittet received a grant from NIGMS during the conduct of the study. The other authors declare that they have no competing interests.

\section{Author details}

${ }^{1}$ Department of Intensive Care Medicine, Onze Lieve Vrouwe Gasthuis and Amsterdam University Medical Centers, Amsterdam, The Netherlands. ${ }^{2}$ Laboratory of Experimental Intensive Care and Anesthesiology of the Amsterdam University Medical Center, Amsterdam, The Netherlands. ${ }^{3}$ Trauma Unit, Department of Surgery, Amsterdam University Medical Center, Amsterdam, The Netherlands. ${ }^{4}$ Trauma Unit, Department of Surgery, Onze Lieve Vrouwe Gasthuis, Amsterdam, The Netherlands. ${ }^{5}$ Department of Anesthesiology and Perioperative Medicine, University of Alabama at Birmingham, Birmingham, AL, USA. ${ }^{6}$ Department of Cardiology, Drexel University College of Medicine, and ANSAR Medical Technologies, Inc., Philadelphia, PA, USA.

Received: 17 October 2020 Accepted: 13 November 2020

Published online: 25 November 2020

\section{References}

1. Emsley HC, Hopkins SJ (2008) Acute ischaemic stroke and infection: recent and emerging concepts. Lancet Neurol 7:341-353

2. Busl KM (2018) Nosocomial Infections in the Neurointensive Care Unit. Neurosurg Clin N Am 29:299-314

3. Wastfelt M, Cao Y, Strom JO (2018) Predictors of post-stroke fever and infections: a systematic review and metaanalysis. BMC Neurol 18:49

4. Vermeij JD, Westendorp WF, Dippel DW, van de Beek D, Nederkoorn PJ (2018) Antibiotic therapy for preventing infections in people with acute stroke. Cochrane Database Syst Rev 1:Cd008530

5. Hoffmann S, Harms H, Ulm L, Nabavi DG, Mackert BM, Schmehl I, Jungehulsing GJ, Montaner J, Bustamante A, Hermans M, Hamilton F, Gohler J, Malzahn U, Malsch C, Heuschmann PU, Meisel C, Meisel A (2017) Stroke-induced immunodepression and dysphagia independently predict stroke-associated pneumonia — the PREDICT study. J Cereb Blood Flow Metab 37:3671-3682

6. Prass K, Meisel C, Hoflich C, Braun J, Halle E, WolfT, Ruscher K, Victorov IV, Priller J, Dirnagl U, Volk HD, Meisel A (2003) Stroke-induced immunodeficiency promotes spontaneous bacterial infections and is mediated by sympathetic activation reversal by poststroke Thelper cell type 1-like immunostimulation. J Exp Med 198:725-736

7. Shim R, Wong CH (2016) Ischemia, Immunosuppression and infection-tackling the predicaments of post-stroke complications. Int J Mol Sci 17:64

8. Santos Samary C, Pelosi P, Leme Silva P, Rieken Macedo Rocco P (2016) Immunomodulation after ischemic stroke: potential mechanisms and implications for therapy. Critical Care (London, England) 20:391

9. Buchman TG, Stein PK, Goldstein B (2002) Heart rate variability in critical illness and critical care. Curr Opin Crit Care 8:311-315

10. Kox M, Vrouwenvelder MQ, Pompe JC, van der Hoeven JG, Pickkers P, Hoedemaekers CW (2012) The effects of brain injury on heart rate variability and the innate immune response in critically ill patients. J Neurotrauma 29:747-755

11. Colombo J, Shoemaker WC, Belzberg H, Hatzakis G, Fathizadeh P, Demetriades D (2008) Noninvasive monitoring of the autonomic nervous system and hemodynamics of patients with blunt and penetrating trauma. J Trauma 65:1364-1373

12. van Vught LA, Klein Klouwenberg PM, Spitoni C, Scicluna BP, Wiewel MA, Horn J, Schultz MJ, Nurnberg P, Bonten MJ, Cremer OL, van der Poll T (2016) Incidence, risk factors, and attributable mortality of secondary infections in the intensive care unit after admission for sepsis. JAMA 315:1469-1479

13. Balvers K, Curry N, Kleinveld DJ, Boing AN, Nieuwland R, Goslings JC, Juffermans NP (2015) Endogenous microparticles drive the proinflammatory host immune response in severely injured trauma patients. Shock (Augusta, Ga) 43:317-321

14. Task Force of the European Society of Cardiology and the North American Society of Pacing and Electrophysiology. Heart ratevariability: standards of measurement, physiological interpretation and clinical use. Circulation 1996;93:1043-65

15. Poole D, Chieregato A, Langer M, Viaggi B, Cingolani E, Malacarne P, Mengoli F, Nardi G, Nascimben E, Riccioni L, Turriziani I, Volpi A, Coniglio C, Gordini G (2014) Systematic review of the literature and evidence-based recommendations for antibiotic prophylaxis in trauma: results from an Italian consensus of experts. PLoS ONE 9:e113676

16. Doran SJ, Henry RJ, Shirey KA, Barrett JP, Ritzel RM, Lai W, Blanco JC, Faden AI, Vogel SN, Loane DJ (2020) Early or late bacterial lung infection increases mortality after traumatic brain injury in male mice and chronically impairs monocyte innate immune function. Crit Care Med 48:e418-e428

17. Lees T, Shad-Kaneez F, Simpson AM, Nassif NT, Lin Y, Lal S (2018) Heart rate variability as a biomarker for predicting stroke, post-stroke complications and functionality. Biomark Insights 13:1177271918786931

18. Hatzakis G, Belzberg H, Shoemaker W, Colombo J, Demetriades D. Noninvasive autonomic and hemodynamic monitoring in severe sepsis. J Clin Anesth Pain Med. 2017;2017:1-9.

19. Borovikova LV, Ivanova S, Zhang M, Yang H, Botchkina Gl, Watkins LR, Wang H, Abumrad N, Eaton JW, Tracey KJ (2000) Vagus nerve stimulation attenuates the systemic inflammatory response to endotoxin. Nature 405:458-462

20. Gunther A, Salzmann I, Nowack S, Schwab M, Surber R, Hoyer H, Witte OW, Hoyer D (2012) Heart rate variability-a potential early marker of sub-acute post-stroke infections. Acta Neurol Scand 126:189-196 
21. Sykora M, Siarnik P, Diedler J (2015) beta-Blockers, pneumonia, and outcome after ischemic stroke: evidence from virtual international stroke trials archive. Stroke 46:1269-1274

22. Maier IL, Becker JC, Leyhe JR, Schnieder M, Behme D, Psychogios MN, Liman J (2018) Influence of beta-blocker therapy on the risk of infections and death in patients at high risk for stroke induced immunodepression. PLOS ONE 13:e0196174

23. Starr JB, Tirschwell DL, Becker KJ (2017) Increased infections with beta-blocker use in ischemic stroke, a beta2-receptor mediated process? Neurol Sci 38:967-974

24. Chen Z, Tang L, Xu X, Wei X, Wen L, Xie Q (2017) Therapeutic effect of beta-blocker in patients with traumatic brain injury: a systematic review and meta-analysis. J Crit Care 41:240-246

25. Aboab J, Mayaud L, Sebille V, de Oliveira R, Jourdain M, Annane D (2018) Esmolol indirectly stimulates vagal nerve activity in endotoxemic pigs. Intensive Care Med Exp 6:14

26. Shin SS, Dixon CE (2015) Alterations in cholinergic pathways and therapeutic strategies targeting cholinergic system after traumatic brain injury. J Neurotrauma 32:1429-1440

27. Engel O, Akyuz L, da Costa Goncalves AC, Winek K, Dames C, Thielke M, Herold S, Bottcher C, Priller J, Volk HD, Dirnagl U, Meisel C, Meisel A (2015) Cholinergic pathway suppresses pulmonary innate immunity facilitating pneumonia after stroke. Stroke 46:3232-3240

28. Lafargue M, Xu L, Carles M, Serve E, Anjum N, lles KE, Xiong X, Giffard R, Pittet JF (2012) Stroke-induced activation of the alpha7 nicotinic receptor increases Pseudomonas aeruginosa lung injury. FASEB J 26:2919-2929

29. Aysin B, Aysin E (2006) Effect of respiration in heart rate variability (HRV) analysis. Conference proceedings: Annual International Conference of the IEEE Engineering in Medicine and Biology Society IEEE Engineering in Medicine and Biology Society Annual Conference 1:1776-1779

\section{Publisher's Note}

Springer Nature remains neutral with regard to jurisdictional claims in published maps and institutional affiliations.

\section{Submit your manuscript to a SpringerOpen ${ }^{\odot}$ journal and benefit from:}

- Convenient online submission

- Rigorous peer review

- Open access: articles freely available online

- High visibility within the field

Retaining the copyright to your article

Submit your next manuscript at $\boldsymbol{\nabla}$ springeropen.com 